AperTO - Archivio Istituzionale Open Access dell'Università di Torino

\title{
3D Thermal Mapping of Architectural Heritage
}

\section{This is the author's manuscript}

Original Citation:

\section{Availability:}

This version is available http://hdl.handle.net/2318/1785304

since 2021-04-13T11:07:54Z

Publisher:

Springer Nature Switzerland AG

Published version:

DOI:10.1007/978-3-030-73043-7_3

Terms of use:

Open Access

Anyone can freely access the full text of works made available as "Open Access". Works made available under a Creative Commons license can be used according to the terms and conditions of said license. Use of all other works requires consent of the right holder (author or publisher) if not exempted from copyright protection by the applicable law. 


\title{
3D Thermal Mapping of Architectural Heritage
}

\section{Up-to-Date Workflows for the Production of Three-Dimensional Thermographic Models for Built Heritage NDT}

\author{
Efstathios Adamopoulos ${ }^{1(*)}[0000-0003-4358-474 X]$, Giacomo Patrucco ${ }^{2[0000-0003-3061-5316]}$, Mo- \\ nica Volinia ${ }^{2}$, Mario Girotto ${ }^{2}$, Fulvio Rinaudo ${ }^{20000-0002-9592-1341]}$, Fabio Giulio Tono- \\ $10^{2[0000-0001-5783-0951]}$ and Antonia Spanò 2,3[0000-0003-4243-7959] \\ ${ }^{1}$ Department of Computer Science, University of Turin, Corso Svizzera 185, Turin 10149, Italy \\ efstathios.adamopouloseunito.it \\ ${ }^{2}$ Department of Architecture and Design, Polytechnic University of Turin, Viale Pier Andrea \\ Mattioli 39, 10125 Turin, Italy \\ \{giacomo.patrucco, monica.volinia, mario.girotto, \\ fulvio.rinaudo, fabio.giuliotonolo, antonia.spano\} epolito.it \\ ${ }^{3}$ PoliTo FULL | the Future Urban Legacy Lab, Toolbox coworking. Via Agostino da Montefel- \\ tro 2, 10125 Turin, Italy
}

\begin{abstract}
The combination of thermographic and geometric recording has always been an issue for architectural heritage diagnostic investigations. Multidisciplinary projects often require integrating multi-sensor informationincluding metric and temperature data - to extract valid conclusions regarding the state-of-preservation of historical buildings. Towards this direction, recent technological advancements in thermographic cameras and three-dimensional (3D) documentation instrumentation and software have contributed significantly, assisting the rapid creation of detailed 3D thermal-textured results, which can be exploited for non-destructive diagnostical surveys. This paper aims to briefly review and evaluate the current workflows for thermographic architectural 3D modeling, which implement state-of-the-art sensing procedures and processing techniques, while also presenting some applications on case studies of significant heritage value to help discuss current problems and identify topics for relevant future research.
\end{abstract}

Keywords: 3D Thermography, Thermal Imaging, Data Integration, NonDestructive Testing, Architectural Heritage, Heritage Conservation.

\section{$1 \quad$ Introduction}

Infrared thermography (IRT) is a consolidated remote sensing technique for the nondestructive assessment of architectural heritage surfaces. Over the last years, the sensors used for the documentation and assessment of thermal phenomena have evolved and diversified. Therefore, IRT has been extensively applied regarding cultural heritage for the evaluation of the state-of-preservation of traditional and historical materials and structures [1], the behavior of replacement materials [2], the effectiveness of

The final authenticated publication is available online at https://doi.org/10.1007/978-3-030-73043-7_3 
the cleaning of architectural surfaces, the efficacy of consolidation interventions, the compatibility of masonry restoration interventions by repair mortars $[3,4]$, the extent of plaster detachment and integration [5], the moisture content [6], as well as for the uncovering of hidden features and subsurface construction $[7,8]$.

Thermographic investigations of architectural heritage are often conducted independently from the acquisition of the structure's geometry and examine thermal phenomena on a local scale by acquiring two-dimensional (2D) thermograms. This is mainly a result of thermal-infrared (TIR) sensors' technical characteristics, such as their low resolution. However, the significance of geometry in the field of heritage diagnostics is high, which has led to various approaches towards the combination of 3D metric surveying and temperature mapping. These integrated geomatics approaches aim to produce either 3D models or 2D derivative orthoimages combined with TIR information, enabling accurate measurements of discontinuity locations and further diagnostical data fusion. The presented work tracks the developments in 3D temperature mapping for architectural heritage investigations by briefly reviewing techniques for 3D shape and TIR data acquisition, and integration, in the relevant literature. Some practical examples from the author's recent work are also presented in the following to evaluate the current state-of-the-art in applicable non-destructive testing (NDT) techniques.

\section{$2 \quad$ Methods and Applications}

Measuring a historical building's or single building components' 3D geometry is the first integral part of accurate thermographic modeling - and accurate documentation in general-which can be performed with different sensors and concerning different purposes $[9,10]$. Contemporary methods for 3D metric surveying widely used for architectural heritage applications include, but are not limited to, terrestrial and UASbased light detection and ranging (LiDAR; active sensing) and digital photogrammetry (passive sensing). The produced metric results can be integrated into various forms and data-types with the TIR images. Nevertheless, the latest methodological developments enable the direct extraction of 3D metric information from thermal imagery itself, employing photogrammetric-based thermographic data acquisition and multiimage processing workflows.

\subsection{LiDAR and Infrared Thermography}

LiDAR approaches use active range sensors to record the 3D position of every point inside the sensor's field-of-view (FOV) along with the intensity of backscattered radiation. They can produce dense or sampled point clouds of an object in considerably less time than passive methods, making them extremely valuable for high-resolution surveys of existing buildings and architectural elements. In thermographic surveys, LiDAR has often been considered as an optimal complement, given its capacity to swiftly provide point clouds that can be textured with the TIR images directly or after 3D model production. In order to perform the thermal texturing, the geometric rela- 
tion between the thermographic images and the 3D geometry of the object has to be known. The position and orientation of the TIR images can be estimated with different methodologies.

When the TIR camera and the LiDAR sensor are not affixed, and their relative position and orientation are not measured during the acquisition phase, the 3D relation between each thermal image and the produced point cloud has to be estimated individually. The foundation for this estimation's solution is based on correspondence features recognition between the point cloud or the derivative documentation products - which contain metric spatial information - and the TIR images. The relative position and orientation-matrix of each TIR image can be estimated using the manual identification of corresponding (homologous) points [11-15]. Figure 1 showcases the result of TIR image-texturing for a partial model of the East façade of Castello del Valentino in Turin (Italy), aiming to identify deterioration patterns, such as detachments, dampness, and biological colonization.

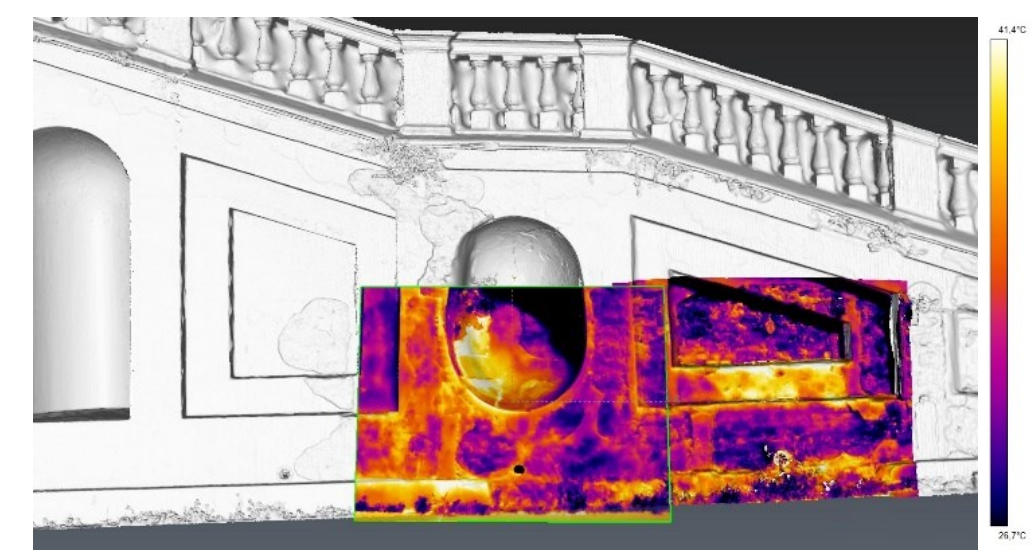

Fig. 1. The thermographic 3D model is generated with the registration of products: the thermal images and the 3D mesh produced with terrestrial LiDAR.

More advanced thermal texturing approaches of the geometric products address the automated matching of $2 \mathrm{D}$ features on the TIR images with the corresponding 3D features on the object's model. Hoegner and Stilla [16] described two workflows for matching infrared photos with 3D building models and 3D point clouds. The first included registering a building model with a sequence of terrestrial thermal images, using geometrical constraints generated from the model's geometry to refine thermal images' orientation. The second workflow used an Iterative Closest Point (ICP) strategy to register an RGB imagery-produced 3D point cloud and a TIR image-based 3D point cloud, to assign thermal values to the dense RGB point cloud. Lagüela et al. [17] and González-Aguilera et al. [18] extracted features from point clouds and LiDAR-produced range images respectively, to facilitate their registration.

Approaches for the simultaneous acquisition of thermal and 3D geometrical data from terrestrial LiDAR - in the form of point clouds - have also been developed for rapid $3 \mathrm{D}$ thermographic mapping. The utilization of commercial multi-sensor solu- 
tions or custom-made instrumentation requires the orientation of the different integrated sensors used for the acquisition. Borrmann et al. [19] built and calibrated an automatic moving setup for the simultaneous acquisition of 3D laser scan data, thermal, and RGB images combining an Optris PI 160 TIR camera, a Riegl VZ-400 laser scanner, and a Logitech QuickCam Pro 9000 webcam, mounted on a modified VolksBot RT 3 platform. They tested the robotic system to perform thermal recording at the Bremen City Hall (Germany) - a historical building of Brick Gothic and Weser Renaissance architecture. Merchán et al. [20] developed a hybrid thermal scanning system employing a Riegl VZ-400 laser scanner, a Nikon D90 color camera, and a FLIR AX5 TIR camera. The hybrid instrument was calibrated with targets incorporating both visible and thermal reflectance discriminants, distributed over a wide area of the scene. It was also used for the Baritel de San Carlos's interior thermal mapping in Almadenejos and the Nuestra Señora de la Candelaria church in Fuente del Maestre (Spain).

\subsection{Single-Thermographic Image Rectification}

In principle, thermographic sensors, such as those used for building inspections, can be handled as standard photogrammetric cameras [21]. As in optical photography, the thermal images are subject to distortion effects. Quite often in architectural photogrammetry, the optical axis of the camera is not vertical to the architectural surface, resulting in distortions of the object on the image. Thus, a photogrammetric singleimage digital processing procedure is required [22] to allow the correct data interpretation by generating rectified thermal images, which can be used directly or for texturing point clouds and reconstructed 3D models. Image rectification is one of the simplest and most economic photogrammetric methods and can take two forms.

1. with known camera parameters and availability of a 3D model of the object

When the internal orientation of the TIR camera is known, the geometry of the acquisition is restored, and the position and external orientation parameters of the camera are needed so that the point positions can be estimated as the intersection of rays from the camera to the surface with the surface known for its geometry. To estimate the camera's external orientation parameters, at least three points with known coordinates on the object are needed. If the digital surface model of the object is available, then the thermal values can be projected on it to create an orthographic view of the object's plane, a so-called orthophoto or orthoimage. An alternative rectification method can be the use of scale bars - instead of points with known coordinatesappropriate for the thermographic approach.

2. without knowledge of camera parameters and almost planar objects

For the application of the rectification process, in this case, a projective transformation is needed between the plane of the thermal image and the object's surface (projective plane). Estimating the transform parameters requires at least four points with known coordinates on the object plane [23]. 


\subsection{Multi-Image 3D Reconstruction and Infrared Thermography}

Multi-image 3D reconstruction approaches constitute a cost-effective alternative to traditional close-range photogrammetry, which can effectively involve both nadiral and oblique imagery to generate accurate and high-resolution metric products. Nowadays, thanks to the improvements in the fields of photogrammetric computer vision technologies and image-matching algorithms, these solutions are characterized by a high level of automatization. As a result, these techniques have become widely applied in architectural heritage recording. However, despite the fact that user-friendly and almost automatic solutions exist, it should be underlined that the role of the operator is still fundamental to ensure that the metric and thematic accuracy of the final products meet the user requirements [24]. Using robust automated Structure-fromMotion (SfM) and Multiple-View-Stereo (MVS) algorithmic implementations, dense $3 \mathrm{D}$ point clouds and models can be generated from properly overlapping images.

\section{Terrestrial Applications}

Recently, few approaches have been reported using multiple-view image-based reconstruction techniques to digitize both $3 \mathrm{D}$ shape and thermal texture using only TIR images for building related-applications (Figure 2) [25,26].

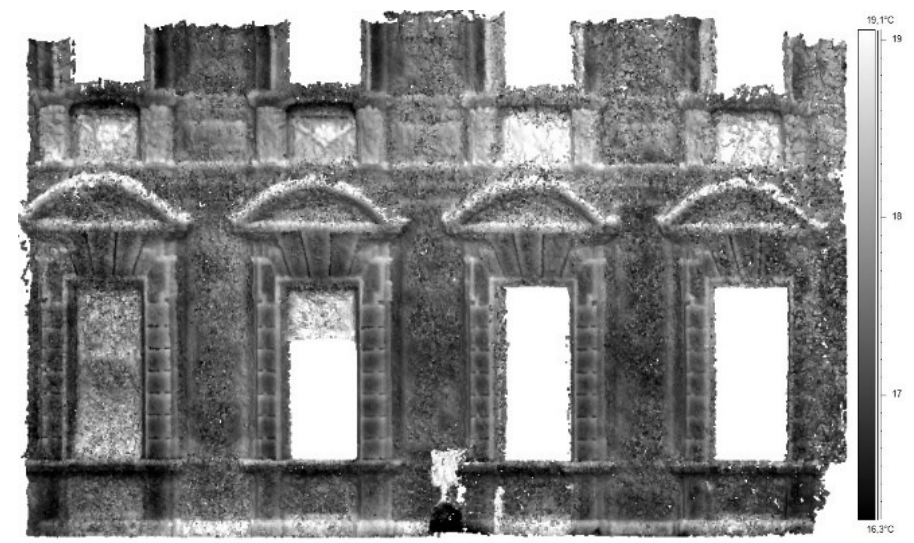

Fig. 2. The thermographic 3D point cloud is generated after the application of photogrammetric principles to thermographic images.

Lagüela et al. [27] highlighted that certain specifications have to be met for the successful generation of 3D point clouds directly from thermographic imagery, such as the acquisition of orthogonal and oblique images, which will be used for the accurate implementation of the photogrammetric principles, maintaining a robust geometry for the reconstruction, and exploitation of only the orthogonal images for texturing $3 \mathrm{D}$ the results - to avoid the inclination and convergence effect.

Due to the inherently different characteristics between TIR and RGB images, research on thermographic modeling for architectural heritage has mainly concentrated on workflows reconstructing $3 \mathrm{D}$ shape from RGB images and applying the texture 
from registered TIR images, and hybrid workflows which apply the photogrammetric principles on both RGB and TIR images and use only the latter for texturing. Previtalli et al. [28] developed an approach to compute photogrammetrically the orientation of both thermal and RGB images together in a combined bundle adjustment (using the collinearity equation) in order to improve co-registration accuracies and map the infrared images on models of building façades. Lin et al. [29] proposed a thermal mapping workflow based on registration between the thermal point cloud and RGB point cloud performed using fast global registration and image resection of TIR images.

Aiming to tackle the problems caused by TIR images' low spatial resolution, we developed a cost-effective thermal mapping method employing optical and IRT terrestrial datasets from a TIR camera and a high-resolution digital RGB camera. The RGB and TIR sensors of the thermographic camera were calibrated to model the image distortions. Then, undistorted TIR images were registered with a projective transformation to match the system of the undistorted RGB images - captured with the optical sensor of the thermographic camera - using the same transformation parameters for each strip of images. The imagery dataset from the high-resolution RGB camera was used to improve and densify the photogrammetric reconstruction produced with RGB images derived from the thermographic camera. After 3D point cloud and final model generation, the oriented RGB images were replaced by the corresponding transformed TIR images to apply the thermal texture (Figure 3). Since the thermographic images were not used for generating the geometric model, a higher number of points was reconstructed, resulting in an accurate higher resolution model. Simultaneously, the spatial resolution of the thermal information was higher due to the large number of used TIR images that were acquired from a close range [30].

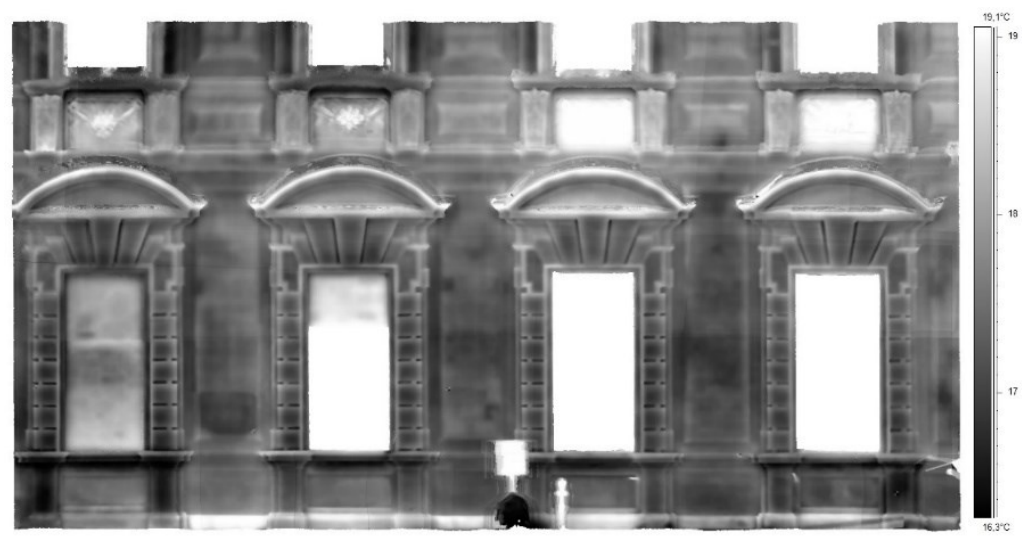

Fig. 3. Model textured with thermal images, generated with the workflow described in 2.3 .

\section{UAS-Based Applications.}

As is well-known, nowadays, UAS (Unmanned Aircraft Systems)-based photogrammetry presents an effective and relatively low-cost solution for cultural heritage metric documentation, allowing a rigorous and accurate geometric reconstruction of the 
built and landscape environment [31]. The developments of new COTS (Commercial Off-the-Shelf) UAS platforms and the improvements in photogrammetric SfM-based algorithms lead to increased and effective use of UAS for mapping purposes in different application domains. Nowadays, specialists in many fields of expertise (including researchers operating in the framework of heritage valorization) are successfully using UAS photogrammetry to carry out their research.

The introduction of COTS drones equipped with high-performing thermal sensors allows researchers in the field of cultural heritage to acquire and analyze thermal data from an aerial perspective - integrating and complementing traditional close-range approaches (with a higher level of detail with respect to manned aerial or satellite platforms). As evidence of the growing interest in this research topic, several commercial and open-source SfM-based software are implementing new algorithms to process thermal data in order to successfully perform 3D reconstruction, including specific templates for UAS acquired thermograms [32,33].

The possibility to process UAS TIR images using SfM-based algorithms allows thermal information and 3D geometry data of the object to be integrated for monitoring, inspection, or diagnostic purposes.

Considering the intrinsic vulnerability of the assets belonging to built heritage, the opportunity represented by using a remote diagnostic tool such as IRT data for photogrammetric applications is particularly interesting for the disciplines connected to documentation and restoration. The low spatial resolution of the thermal images can impose a severe obstacle to the proper reconstruction of the surveyed structures' geometry. Moreover, the radiometric characteristics of TIR images make the direct use of SfM challenging [32]: for this reason, some critical issues are generally encountered during the tie-points extraction phase [34], especially if the acquisition has been performed without following rigorous photogrammetric overlapping criteria.

In the case of UAS based acquisitions, as underlined in the previous section, a multi-sensor strategy can represent a valid solution to avoid topological errors during the 3D reconstruction phase - which could cause some projection miscalculations during orthoimagery production - and to achieve better results in terms of the geometric and spatial resolution of the final model $[35,36]$. Following this strategy, and by integrating thermal and optical images, it is possible to exploit the higher geometric and spatial resolution of the traditional true-color images to generate a more detailed, accurate, and topologically correct 3D mesh, which can be used as a geometric reference surface both for the texturization process of the achieved 3D model with IRT data and the generation of thermal orthoimagery. This approach requires the registration of both thermal and optical images in the same reference system, which is based on the identification of the Ground Control Points (GCPs) also in thermal images, a challenging task due to the intrinsic characteristics of this kind of data. This problem can be solved using the image geo-tag [34], which is often embedded in the acquired images (if the system used is equipped with a GNSS receiver). However, this solution's positional accuracy is generally low (few meters) unless RTK-enabled platforms are adopted [37]. 

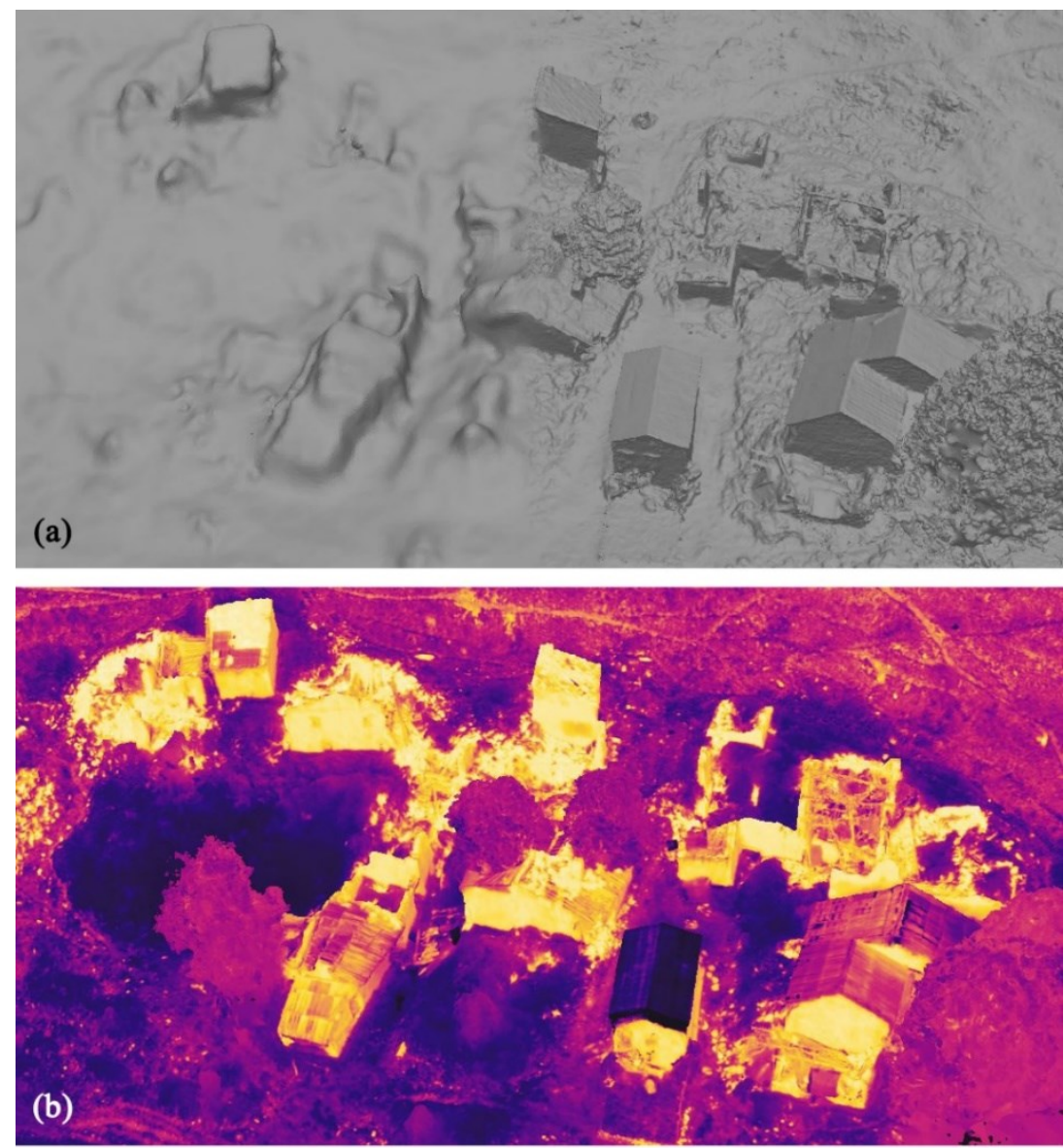

$10.5^{\circ} \mathrm{C}$

$39.40^{\circ} \mathrm{C}$

Fig. 4. (a) Level of detail differences between the mesh obtained from thermal dataset only (on the left) and optical dataset (on the right). (b) 3D mesh obtained from optical dataset texturized with co-registered thermal images.

A practical solution consists of using specific artificial targets made of materials characterized by a low emissivity, in contrast to the traditional photogrammetric targets (usually made of paper or plastic, which are characterized by an emissivity value up to 0.99). This solution has been used in Hill et al. 2020 [38], where aluminum targets have been used to detect them easily and unambiguously in TIR images.

Lastly, a third workflow-which has been used in Patrucco el al. 2020 [35] in the context of an experience on an abandoned alpine hamlet subject (in the framework of research on the regeneration of small alpine settlements) - assesses the possibility to use natural points as control points, when clearly detectable on both optical and thermal images. 
As is possible to observe in Figure 5, following a traditional SfM-based workflow and using the aforementioned approaches, it is feasible to generate high resolution and high detailed 3D models with a thermal texture or generate thermal orthoimagery.

\section{Discussion and Conclusions}

The fusion of geomatics and thermographic techniques has a great added value for the implementation of thermographic surveys for historical architecture. This review presented several methods focused on this application domain, including techniques for simultaneous or independent acquisition of geometric and thermal information, as well as state-of-the-art workflows employing SfM/MVS-based approaches. The results showcase the potential for combined non-destructive evaluation of the state-ofpreservation of built heritage.

The registration of single thermograms to LiDAR or photogrammetry-produced point clouds and 3D models is a simple-to-implement method to investigate local phenomena. The generation of extensive thermal orthophoto-maps with this method may prove time-consuming if corresponding features have to be separately computed for each image. Additionally, feature correspondences may not be visible between IRT data and optical imagery, making the registration unfeasible without placing any artificial targets detectable in both the visible and infrared spectra. However, the implementation of automated feature matching techniques can make the methodology more efficient. A significant drawback for product registration is that the relative planarity of the architectural façade or element is required so that occlusions and other geometrical irregularities will not affect the thermal texturing.

Single TIR-image rectification has proven to be a cost-effective method to create spatially correct 2D thermal products. It maximizes the potential for radiometric and spatial measurements, which can be obtained by a single thermal photo after appropriate corrections have been performed. However, it requires calibration of the thermal sensor and, as with product registration, can be used to study only localized phenomena with sufficient spatial resolution.

The application of multi-image 3D reconstruction principles to exploit the geometrical data directly captured with TIR images is a cost-effective approach for rapid 3D diagnostics, but the thermograms' low-resolution significantly restricts the resolution of spatial results. However, hybrid methods involving both RGB and TIR images can produce extensive and even full-building 3D models in high resolution. This either involves RGB and TIR images' simultaneous orientation in the same photogrammetric dataset to acquire accurate orientation information or image registration for accurate thermal texturing of high-resolution reconstructed 3D products from RGB images for terrestrial and aerial applications both. The ability of contemporary $3 \mathrm{D}$ temperature mapping approaches to produce high-resolution results reveals an excellent potential for holistic multidisciplinary and multi-sensor approaches towards protecting cultural heritage. 


\section{Acknowledgments}

This project has partially received funding from the European Union's Framework Program for Research and Innovation Horizon 2020 (2014-2020) under the MarieSkłodowska Curie Grant (Agreement 754511) and from the Compagnia di San Paolo.

\section{References}

1. Grinzato, E., Bison, P.G., Marinetti, S.: Monitoring of ancient buildings by the thermal method. Journal of Cultural Heritage. 3, 21-29 (2002). https://doi.org/10.1016/S12962074(02)01159-7.

2. Lerma, C., Mas, Á., Gil, E., Vercher, J., Torner, M.E.: Quantitative Analysis Procedure for Building Materials in Historic Buildings by Applying Infrared Thermography. Russ J Nondestruct Test. 54, 601-609 (2018). https://doi.org/10.1134/S1061830918080065.

3. Avdelidis, N.P., Moropoulou, A.: Applications of infrared thermography for the investigation of historic structures. Journal of Cultural Heritage. 5, 119-127 (2004). https://doi.org/10.1016/j.culher.2003.07.002.

4. Moropoulou, A., Labropoulos, K.C., Delegou, E.T., Karoglou, M., Bakolas, A.: Nondestructive techniques as a tool for the protection of built cultural heritage. Construction

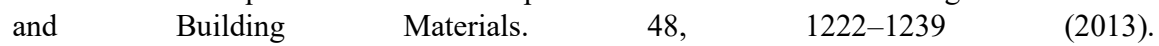
https://doi.org/10.1016/j.conbuildmat.2013.03.044.

5. Volinia, M.: Integration of qualitative and quantitative infrared surveys to study the plaster conditions of Valentino Castle. Presented at the AeroSense 2000, Orlando, FL March 30 (2000). https://doi.org/10.1117/12.381566.

6. Lerma, J.L., Cabrelles, M., Portalés, C.: Multitemporal thermal analysis to detect moisture on a building façade. Construction and Building Materials. 25, 2190-2197 (2011). https://doi.org/10.1016/j.conbuildmat.2010.10.007.

7. Brooke, C.: Thermal Imaging for the Archaeological Investigation of Historic Buildings. Remote Sensing. 10, 1401 (2018). https://doi.org/10.3390/rs10091401.

8. Glavaš, H., Hadzima-Nyarko, M., Buljan, I.H., Barić, T.: Locating Hidden Elements in Walls of Cultural Heritage Buildings by Using Infrared Thermography. Buildings. 9, 32 (2019). https://doi.org/10.3390/buildings9020032.

9. Georgopoulos, A.: Data Acquisition for the Geometric Documentation of Cultural Heritage. In: Ioannides, M., Magnenat-Thalmann, N., and Papagiannakis, G. (eds.) Mixed Reality and Gamification for Cultural Heritage. pp. 29-73. Springer International Publishing, Cham (2017). https://doi.org/10.1007/978-3-319-49607-8_2.

10. Chiabrando, F., Sammartano, G., Spanò, A., Spreafico, A.: Hybrid 3D Models: When Geomatics Innovations Meet Extensive Built Heritage Complexes. IJGI. 8, 124 (2019). https://doi.org/10.3390/ijgi8030124.

11. Costanzo, A., Minasi, M., Casula, G., Musacchio, M., Buongiorno, M.: Combined Use of Terrestrial Laser Scanning and IR Thermography Applied to a Historical Building. Sensors. 15, 194-213 (2014). https://doi.org/10.3390/s150100194.

12. Lerma, J.L., Cabrelles, M., Portalés, C.: Multitemporal thermal analysis to detect moisture on a building façade. Construction and Building Materials. 25, 2190-2197 (2011). https://doi.org/10.1016/j.conbuildmat.2010.10.007.

13. Mileto, C., Vegas, F., Lerma, J.L.: Multidisciplinary Studies, Crossreading and Transversal Use of Thermography: The Castle of Monzón (Huesca) as a case study. In: Editorial 
Universitat Politècnica de València (ed.) Modern Age Fortifications of the Mediterranean Coast - Defensive architecture of the Mediterranean (FORTMED2015). UPV Press, Valencia (2015). https://doi.org/10.4995/FORTMED2015.2015.1786.

14. Spanò, A., Volinia, M., Girotto, M.: Spatial Data and Temperature: Relationship to Deepen. Integrated Methods for Advanced Architectural Diagnosis and Metric Documentation. In: Marabelli, M., Parisi, C., Buzzanca, G., Paradisi, A., (eds.) $8^{\text {th }}$ International Conference on Non Destructive Investigations and Microanalysis for the Diagnostics and Conservation of the Cultural and Environmental Heritage, pp. 405-412. Italian Society for NonDestructive Testing Monitoring Diagnostics AIPnD, Brescia (2005).

15. Zalama, E., Gómez-García-Bermejo, J., Llamas, J., Medina, R.: An Effective Texture Mapping Approach for 3D Models Obtained from Laser Scanner Data to Building Documentation: An effective texture mapping approach. Computer-Aided Civil and Infrastructure Engineering. 26, 381-392 (2011). https://doi.org/10.1111/j.1467-8667.2010.00699.x.

16. Hoegner, L., Stilla, U.: Mobile thermal mapping for matching of infrared images with 3D building models and 3D point clouds. Quantitative InfraRed Thermography Journal. 1-19 (2018). https://doi.org/10.1080/17686733.2018.1455129.

17. Lagüela, S., Díaz-Vilariño, L., Martínez, J., Armesto, J.: Automatic thermographic and RGB texture of as-built BIM for energy rehabilitation purposes. Automation in Construction. 31, 230-240 (2013). https://doi.org/10.1016/j.autcon.2012.12.013.

18. González-Aguilera, D., Rodriguez-Gonzalvez, P., Armesto, J., Lagüela, S.: Novel approach to 3D thermography and energy efficiency evaluation. Energy and Buildings. 54, 436-443 (2012). https://doi.org/10.1016/j.enbuild.2012.07.023.

19. Borrmann, D., Elseberg, J., Nüchter, A.: Thermal 3D Mapping of Building Façades. In: Lee, S., Cho, H., Yoon, K.-J., and Lee, J. (eds.) Intelligent Autonomous Systems 12. pp. 173-182. Springer Berlin Heidelberg, Berlin, Heidelberg (2013). https://doi.org/10.1007/978-3-642-33926-4_16.

20. Merchán, P., Merchán, M.J., Salamanca, S., Adán, A.: Application of Multisensory Technology for Resolution of Problems in the Field of Research and Preservation of Cultural Heritage. In: Ioannides, M., Martins, J., Žarnić, R., and Lim, V. (eds.) Advances in Digital Cultural Heritage. pp. 32-47. Springer International Publishing, Cham (2018). https://doi.org/10.1007/978-3-319-75789-6_3.

21. Luhmann, T., Piechel, J., Roelfs, T.: Geometric Calibration of Thermographic Cameras. In: Kuenzer, C. and Dech, S. (eds.) Thermal Infrared Remote Sensing. pp. 27-42. Springer Netherlands, Dordrecht (2013). https://doi.org/10.1007/978-94-007-6639-6_2.

22. Franzen, C., Siedler, G., Franzen, C., Vetter, S.: Orthogonal IRT imaging. In: 2013 Digital Heritage International Congress (DigitalHeritage). pp. 633-636. IEEE, Marseille, France (2013). https://doi.org/10.1109/DigitalHeritage.2013.6743805.

23. Hemmleb, M., Wiedemann, A.: Digital rectification and generation of orthoimages in architectural photogrammetry. Int. Arch. Photogramm. Remote Sens. Spatial Inf. Sci. XXXII-5C1B-1997, 261-267 (1997).

24. Santagati, C., Inzerillo, L., Di Paola, F.: Image-based modeling techniques for architectural heritage 3D digitalization: Limits and potentialities. Int. Arch. Photogramm. Remote Sens. Spatial Inf. Sci. XL-5/W2, 555-560 (2013). https://doi.org/10.5194/isprsarchives-XL-5W2-555-2013.

25. González-Aguilera, D., Lagüela, S., Rodríguez-Gonzálvez, P., Hernández-López, D.: Image-based thermographic modeling for assessing energy efficiency of buildings façades. Energy and Buildings. 65, 29-36 (2013). https://doi.org/10.1016/j.enbuild.2013.05.040. 
26. Dlesk, A., Vach, K., Holubec, P.: Usage of photogrammetric processing of thermal images for civil engineers. Int. Arch. Photogramm. Remote Sens. Spatial Inf. Sci. XLII-5, 99-103 (2018). https://doi.org/10.5194/isprs-archives-XLII-5-99-2018.

27. Lagüela, S., Díaz-Vilariño, L., Roca, D., Filgueira, A.: In: Riveiro, B., Solla, M. (eds.) Non-Destructive Techniques for the Evaluation of Structures and Infrastructure. pp. 233252. CRC Press (2016). https://doi.org/10.1201/b19024.

28. Previtali, M., Barazzetti, L., Redaelli, V., Scaioni, M., Rosina, E.: Rigorous procedure for mapping thermal infrared images on three-dimensional models of building façades. J. Appl. Remote Sens. 7, 073503 (2013). https://doi.org/10.1117/1.JRS.7.073503.

29. Lin, D., Jarzabek-Rychard, M., Tong, X., Maas, H.-G.: Fusion of thermal imagery with point clouds for building façade thermal attribute mapping. ISPRS Journal of Photogrammetry and Remote Sensing. 151, 162-175 (2019). https://doi.org/10.1016/j.isprsjprs.2019.03.010.

30. Adamopoulos, E., Volinia, M., Girotto, M., Rinaudo, F.: Three-Dimensional Thermal Mapping from IRT Images for Rapid Architectural Heritage NDT. Buildings. 10, 187 (2020). https://doi.org/10.3390/buildings 10100187.

31. Fernández-Hernandez, J., González-Aguilera, D., Rodríguez-Gonzálvez, P., Mancera Taboada, J.: Image-based modelling from unmanned aerial vehicle (UAV) photogrammetry: an effective, low-cost tool for archaeological applications. Archaeometry, 57(1), 128145 (2015). https://doi.org/10.1111/arcm.12078

32. Javadnejad, F., Gillins, D. T., Parrish, C. E., Slocum, R. K.: A photogrammetric approach to fusing natural colour and thermal infrared UAS imagery in 3D point cloud generation. Inter-national Journal of Remote Sensing. 41(1), 211-237 (2019). https://doi.org/10.1080/01431161.2019.1641241

33. Gonzalez-Aguilera, D., López Fernández, L., RodriguezGonzalvez, P., Hernandez-Lopez, D., Guerrero, D., Remondino, F., Menna, F., Nocerino, E., Toschi, I., Ballabeni, A., Gaiani, M.: GRAPHOS - Open-source software for photogrammetric applications. The Photogrammetric Record, 33(161), 11-29 (2018). https://doi.org/10.1111/phor.12231

34. Jarząbek-Rychard, M., Lin, D., Maas, H. G.: Supervised detection of façade openings in 3D point clouds with thermal attributes. Remote Sensing, 12(3), 543 (2020). https://doi.org/10.3390/rs12030543

35. Patrucco, G., Cortese, G., Giulio Tonolo, F., Spanò, A.: Thermal and optical data fusion supporting built heritage analyses. Int. Arch. Photogramm. Remote Sens. Spatial Inf. Sci. XLIII-B3， 619-626 (2020). https://doi.org/10.5194/isprs-archives-XLIII-B3-2020-6192020

36. Wakeford, Z. E., Chmielewska, M., Hole, M. J., Howell, J.A.: Combining thermal imaging with photogrammetry of an active volcano using UAV: an example from Stromboli, Italy. The Photogrammetric Record, 34(168), 445-466 (2019). https://doi.org/10.1111/phor.12301

37. Sammartano, G., Chiabrando, F., Spanò, A.: Oblique images and direct photogrammetry with a fixed wing platform: first test and results in Hierapolis of Phrygia (TK). Int. Arch. Photogramm. Remote Sens. Spatial Inf. Sci. XLIII-B2, 75-82 (2020). https://doi.org/10.5194/isprs-archives-XLIII-B2-2020-75-2020

38. Hill, A. C., Laugier, E. J., Casana, J.: Archaeological Remote Sensing Using MultiTemporal, Drone-Acquired Thermal and Near Infrared (NIR) Imagery: A Case Study at the Enfield Shaker Village, New Hampshire. Remote Sens. 12(4), 690 (2020). https://doi.org/10.3390/rs12040690 